\title{
ARTICLE
}

\section{Aggravated ulcerative colitis caused by intestinal Metrnl deficiency is associated with reduced autophagy in epithelial cells}

\author{
Sai-long Zhang ${ }^{1}$, Zhi-yong $\mathrm{Li}^{1}$, Dong-sheng Wang ${ }^{1}$, Tian-ying $\mathrm{Xu}^{1}$, Mao-bing Fan ${ }^{1}$, Ming-he Cheng ${ }^{1}$ and $\mathrm{Chao}$-yu Miao ${ }^{1}$
}

\begin{abstract}
Metrnl is a newly identified secreted protein highly expressed in the intestinal epithelium. This study aimed to explore the role and mechanism of intestinal epithelial Metrnl in ulcerative colitis. Metrnl ${ }^{-1-}$ (intestinal epithelial cell-specific Metrnl knockout) mice did not display any phenotypes of colitis under basal conditions. However, under administration of 3\% dextran sodium sulfate (DSS) drinking water, colitis was more severe in Metrnl $^{-1-}$ mice than in WT mice, as indicated by comparisons of body weight loss, the presence of occult or gross blood per rectum, stool consistency, shrinkage in the colon, intestinal damage, and serum levels of inflammatory factors. DSS-induced colitis activated autophagy in the colon. This activation was partially inhibited by intestinal epithelial Metrnl deficiency, as indicated by a decrease in Beclin-1 and LC3-II/I and an increase in p62 in DSS-treated Metrnl ${ }^{-/-}$mice $^{-1}$ compared with WT mice. These phenomena were further confirmed by observation of autophagosomes and immunofluorescence staining for LC3 in epithelial cells. The autophagy-related AMPK-mTOR-p70S6K pathway was also activated in DSS-induced colitis, and this pathway was partially blocked by intestinal epithelial Metrnl deficiency, as indicated by a decrease in AMPK phosphorylation and an increase in mTOR and p70S6K phosphorylation in DSS-treated Metrnl ${ }^{-1-}$ mice compared with WT mice. Therefore, Metrnl deficiency deteriorated ulcerative colitis at least partially through inhibition of autophagy via the AMPK-mTORp70S6K pathway, suggesting that Metrnl is a therapeutic target for ulcerative colitis.
\end{abstract}

Keywords: Metrnl; ulcerative colitis; autophagy; AMPK-mTOR-p70S6K pathway

Acta Pharmacologica Sinica (2020) 41:763-770; https://doi.org/10.1038/s41401-019-0343-4

\section{INTRODUCTION}

Ulcerative colitis is a type of inflammatory bowel disease. In recent years, research on its etiology and pathogenesis has received extensive attention [1-3]. For the treatment of ulcerative colitis, surgery, anti-infection agents, glucocorticoids, and immunosuppressive agents are often used. However, these are symptomatic treatments, and long-term side effects are likely to cause disease recurrence $[4,5]$.

Autophagy is an important self-protection mechanism and is a cellular metabolic pathway that relies on lysosomes. Previous studies have shown that autophagy plays a protective role in a variety of diseases, including cancer and inflammatory and immune diseases [6-8]. In recent years, it has been reported that there is a close relationship between autophagy and inflammation, especially with certain anti-inflammatory effects [9-11]. In addition, it has been reported that activation of autophagy can ameliorate intestinal inflammation; thus, the induction of autophagy is expected to become a new strategy for the treatment of ulcerative colitis [12].

Metrnl, which is also known as Cometin or Subfatin, is a newly discovered secreted protein containing 311 amino acids with a $\mathrm{NH}_{2}$-terminal signal peptide of 45 amino acids [13]. Our previous study has shown that Metrnl is a new adipokine antagonizing insulin resistance through the PPARY signaling pathway [14, 15]. Metrnl in adipose tissue can promote adipocyte differentiation, improve metabolism, inhibit inflammation, regulate fat function, and alleviate insulin resistance caused by obesity $[13,14,16]$. Through the examination of Metrnl expression in various tissues, we have found that Metrnl is highly expressed in both human and mouse gastrointestinal tissues, especially in intestinal epithelial cells [17]. A recent study has demonstrated that the Metrnl adipokine ameliorates spontaneous colitis in IL-10 knockout mice by attenuating mesenteric adipose tissue lesions [18]. Due to the high expression of Metrnl in intestinal epithelial cells, we speculate that intestinal epithelial Metrnl plays a role in the regulation of ulcerative colitis development.

In the present study, using a conditional knockout mouse model with a specific knockout of Metrnl in intestinal epithelial cells $\left(\mathrm{Metrnl}^{-/-}\right)$, we explored the role of intestinal epithelial Metrnl in the development of dextran sodium sulfate (DSS)induced colitis, an accepted experimental model of ulcerative colitis. We also examined the possible involvement of autophagy in the role of Metrnl.

\footnotetext{
${ }^{1}$ Department of Pharmacology, Second Military Medical University/Naval Medical University, Shanghai 200433, China

Correspondence: Chao-yu Miao (cymiao@smmu.edu.cn)

These authors contributed equally: Sai-long Zhang, Zhi-yong Li
}

Received: 13 June 2019 Accepted: 2 December 2019

Published online: 16 January 2020 
Table 1. Primer sets used in the study.

\begin{tabular}{lll}
\hline & Forward primer $\left(5^{\prime}-3^{\prime}\right)$ & Reversed primer $\left(5^{\prime}-3^{\prime}\right)$ \\
\hline Genes for genotyping & & \\
$\quad$ Floxed Metrnl (Metrnl ${ }^{\text {loxp/loxp }}$ ) & & GGATGAGCGTTTGAGCACAGC \\
Cre (Villin-Cre) & TGAGGGTTGGAGGCTCCTAGC \\
Genes for real-time PCR & GCGGTCTGGCAGTAAAAACTATC & GTGAAACAGCATTGCTGTCACTT \\
Metrnl & & \\
GAPDH & CTGGAGCAGGGAGGCTTATTT & GGACAACAAAGTCACTGGTACAG \\
\hline
\end{tabular}

\section{MATERIALS AND METHODS}

Generation of intestinal epithelial cell-specific Metrnl knockout mice

All animal experiments were performed in accordance with the National Institutes of Health Guide for the Care and Use of Laboratory Animals and were approved by the Animal Ethical Committee of the Second Military Medical University. Metrnl loxP/loxP mice were generated as we described elsewhere [14]. Villin-cre mice [B6. Cg-Tg(Vil1-cre)1000Gum/J; JAX stock 021504] were used to generate intestinal epithelial cell-specific Metrnl knockout $\left(\mathrm{Metrnl}^{-/-}\right.$) mice. The breeding strategy for Metrnl ${ }^{-1-}$ mice was described elsewhere [17]. The littermate Metrnl ${ }^{\text {loxP/loxP }}$ mice were used as the corresponding wild-type (WT) controls.

Cell culture and treatment

Caco- 2 cells were provided by the Cell Bank of Chinese Academy of Sciences (Shanghai, China) and cultured in Dulbecco's modified Eagle's medium with $10 \%$ fetal bovine serum. For certain groups, lipopolysaccharide (LPS) $(10 \mathrm{ng} / \mathrm{mL}$, Sigma-Aldrich, St. Louis, MO, USA) and/or chloroquine (CQ) $(10 \mu \mathrm{M}$, Sigma-Aldrich) were administered for $12 \mathrm{~h}$ for stimulation.

Lentivirus-mediated knockdown of Metrnl in cells Lentiviruses encoding small hairpin RNA (shRNA) directed against human Metrnl were constructed by Shanghai Bio-Link Company (Shanghai, China). Briefly, the following three shRNA target sequences were designed: 5'-GGGCGCTCATTGTTAACCT-3', 5'-GC TTCCAGTATGAGCTGATGA-3', and 5'-CACGCTITAGTGACTTCAA $A-3^{\prime}$. Both the recombinant lentivirus encoding shRNA targeting Metrnl and the lentivirus encoding scrambled shRNA were prepared and titered to $10^{8}$ transfection units $(\mathrm{TU}) / \mathrm{mL}$. The knockdown efficiencies of the three target sequences were assessed in Caco- 2 cells with real-time PCR. The last target sequence displayed the highest knockdown efficiency and was used in the present study [14].

Quantification of mRNA by real-time PCR

Total RNA was extracted from various tissues using TRIzol (Invitrogen, Carlsbad, CA, USA), and real-time PCR was performed using an $A B I 7500$ real-time PCR System (Applied Biosystems) as previously described [15]. A final $20 \mu \mathrm{L}$ reaction mixture included $10 \mu \mathrm{L}$ SYBR Green, $2 \mu \mathrm{L}$ CDNA template, and $1 \mu \mathrm{L}$ primers. The average threshold cycle (CT) was determined from duplicate reactions, the target gene expression was normalized to GAPDH, and the quantitative measurements were obtained using the $\triangle \triangle C T$ method. All primers are listed with their sequences in Table 1.

Histological staining

Hematoxylin and eosin (H\&E) staining was performed as previously described [19]. Briefly, the intestine samples used for histological analysis were fixed with $4 \%$ phosphate-buffered paraformaldehyde for $24 \mathrm{~h}$ and embedded in paraffin. Sections with a thickness of $4 \mu \mathrm{m}$ were prepared and stained with $\mathrm{H} \& \mathrm{E}$.

\begin{tabular}{|lc|}
\hline Table 2. Primary antibodies used in immunofluorescence staining. \\
\hline Antibody & Dilution \\
\hline LC3 (Novus Biologicals) & $1: 200$ \\
CD326 (eBioscience) & $1: 200$ \\
\hline
\end{tabular}

Immunofluorescence staining

The colon was immersed in $4 \%$ paraformaldehyde at $4{ }^{\circ} \mathrm{C}$ for $24 \mathrm{~h}$, cryoprotected in 15\%-30\% (wt/vol) paraformaldehyde, and cut into frozen coronal slices (5- $\mu \mathrm{m}$ thickness) in cryostat (CM3050S; Leica Microsystems, Bannockburn, IL, USA). Colon sections were washed with $1 \times$ PBS three times for $5 \mathrm{~min}$ each. Then, $0.2 \%$ (wt/vol) Triton $\mathrm{X}-100$ and blocking serum were added successively and incubated for $15 \mathrm{~min}$ and $2 \mathrm{~h}$, respectively. The specific primary antibodies were incubated at $4{ }^{\circ} \mathrm{C}$ overnight (Table 2). After being washed three times with $1 \times \mathrm{PBS}$, the sections were incubated with the corresponding secondary antibody (Alexa 488-conjugated and Cy3conjugated) (Jackson Immuno Research Inc., West Grove, PA, USA) for $2 \mathrm{~h}$ at room temperature and protected from light. Nuclei were stained with DAPI (Vector Laboratories, Burlingame, CA, USA) for 10 min. Fluorescence of colon sections was examined under a FLUOVIEW FV1000 confocal laser scanning microscope (Olympus, Japan).

Immunoblot analysis

Protein extraction and immunoblotting were performed as described previously [20]. Briefly, proteins were extracted from the tissue using a standard extraction reagent supplemented with a protease inhibitor (KANGCHEN; Shanghai, China). Protein concentrations were determined using a BCA protein assay kit (Beyotime Institute of Biotechnology; Haimen, China). Proteins were separated using SDSPAGE, electrotransferred to nitrocellulose membranes and incubated with a primary antibody for $8-12 \mathrm{~h}$ at $4{ }^{\circ} \mathrm{C}$ (Table 3 ). The samples were then incubated with an IRDye800CW-conjugated secondary antibody (Rockland; Gilbertsville, PA, USA) for $1 \mathrm{~h}$ at $25^{\circ} \mathrm{C}$. Images were acquired with the Odyssey infrared imaging system (Li-Cor Bioscience; Lincoln, NE, USA). All immunoblotting experiments were repeated at least three times.

Enzyme-linked immunosorbent assay (ELISA)

Serum samples were obtained as previously described [21]. The levels of IL-1 $\beta, I L-6$, and TNF- $\alpha$ in serum samples were quantified using commercial ELISA kits (R\&D system, New York, NY, USA).

Transmission electron microscopy

Colonic tissues were separated and fixed overnight at $4{ }^{\circ} \mathrm{C}$ in $2.5 \%$ glutaraldehyde in $0.1 \mathrm{M}$ PBS and then post fixed in $1 \%$ buffered osmium tetroxide for $2 \mathrm{~h}$. Specimens were processed using routine procedures and examined under a transmission electron microscope (H-700; Hitachi, Tokyo, Japan). 
Induction of colitis in mice

An inflammatory bowel disease model was induced in WT and Metrnl $^{-1-}$ mice with $3 \%$ or $1 \%$ DSS (molecular weight from 36,000 to $50,000 \mathrm{kDa}$, MP Biomedicals LLC, Santa Ana, CA, USA) dissolved in drinking water given ad libitum (1-10 days) as previously described [22].

Disease activity index and histological analysis in mice Body weight, the presence of occult or gross blood per rectum, stool consistency, and colon length were determined by two investigators blinded to the treatment groups. The disease activity index was determined by combining the scores of (i) body weight loss, (ii) stool inconsistency, and (iii) blood in the stool and dividing by 3 [23]. Body weight changes are shown as a loss of baseline body weight. Postmortem, the colon was removed, and pieces of colonic tissue were used for ex vivo analysis. For histology, rings of certain parts of the colon were fixed in $4 \%$ buffered formalin and embedded in paraffin. Sections were stained with $H \& E$ according to standard protocols. Histological scoring was performed by a pathologist in a blinded way. A focally increased number of inflammatory cells in the

\begin{tabular}{|lll|}
\hline Table 3. & Primary antibodies used in Western blots. & \\
\hline Antibody & Molecular weight (kDa) & Dilution \\
\hline Beclin-1 (Cell Signaling Technology) & 60 & $1: 500$ \\
LC3 (Novus Biologicals) & $14 / 16$ & $1: 500$ \\
p62 (Cell Signaling Technology) & 62 & $1: 500$ \\
p-AMPK (Thr172) (Cell Signaling & 62 & $1: 500$ \\
Technology) & 62 & $1: 500$ \\
AMPK (Cell Signaling Technology) & 62 & $1: 500$ \\
p-mTOR (Ser 2448) (Cell Signaling & 289 & $1: 500$ \\
Technology) & 289 & $1: 500$ \\
mTOR (Cell Signaling Technology) & 70 & $1: 500$ \\
p-p70S6K (Ser371) (Cell Signaling & & $1: 1000$ \\
Technology) & 70 & \\
p70S6K (Cell Signaling Technology) & 36 & \\
GAPDH (Beyotime Biotechnology) & & \\
\hline
\end{tabular}

lamina propria was scored as 1 , a confluence of inflammatory cells extending into the submucosa as 2 and a transmural extension of the infiltrate as 3 . For tissue damage, discrete lymphoepithelial lesions were scored as 1 , mucosal erosions as 2, and extensive mucosal damage and/or extension through deeper structures of the bowel wall as 3. Two equally weighted subscores (cell infiltration and tissue damage) were added, and the combined histological colitis severity score ranged from 0 to 6 .

Statistical analysis

All data are presented as the mean \pm SEM and were analyzed using Prism 6.0 software (GraphPad Software). Statistical significance was determined by a two-tailed Student's $t$ test. $P<0.05$ was considered statistically significant.

\section{RESULTS}

Metrnl $^{-/-}$mice exhibit no phenotypes of colitis

We developed intestinal epithelial cell-specific Metrnl knockout (Metrnl ${ }^{-1-}$ ) mice and tested the expression of Metrnl in various types of tissues. The results showed that the expression of Metrnl in Metrnl $^{-1-}$ mice was almost deficient in the colon and small intestine tissues, without significant changes in other examined organs (Fig. 1a). H\&E staining of colon sections showed no significant morphological differences between $\mathrm{Metrnl}^{-/-}$and WT mice (Fig. 1b). To further verify whether intestinal inflammation is induced after the conditional knockout of Metrnl, we performed an assay for inflammatory factors in the colon. The results showed no differences in IL-4, IL-6, and TNF-a between MetrnI ${ }^{-/-}$and WT mice (Fig. 1c, d). These results indicate that colitis is not present after intestinal epithelial deficiency of Metrnl alone.

Three percent DSS drinking water induces colitis on the 5th day Before establishing the DSS-induced colitis model, to choose the optimal observation time and DSS administration concentration, we gave $3 \%$ DSS and $1 \%$ DSS drinking water to WT mice and observed the survival time of these mice at these two DSS concentrations. Fig. 2a shows that one mouse died on day 6 in the
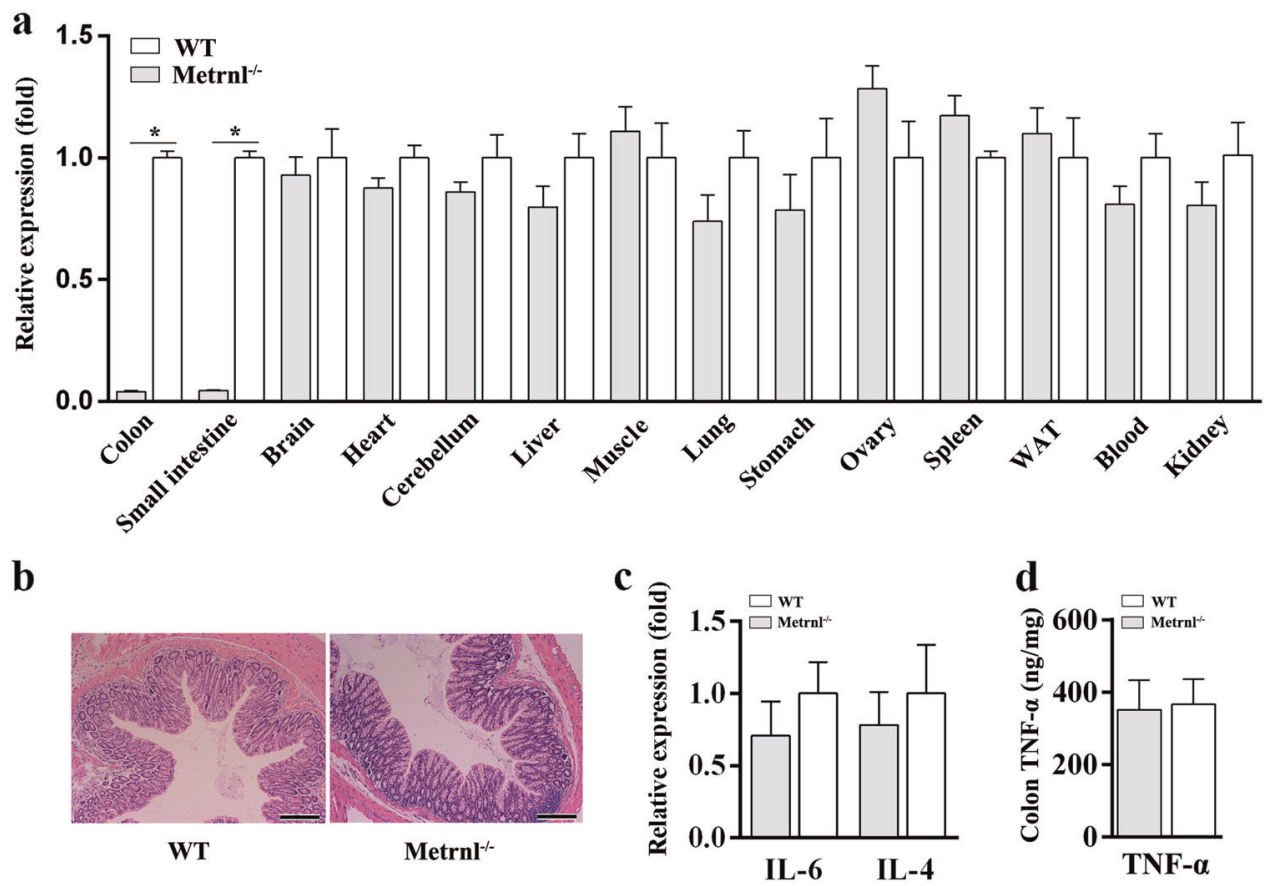

Fig. 1 Metrnl ${ }^{\text {loxP/loxP }}$ Villin-cre (MetrnI ${ }^{-I-}$ ) mice display Metrnl deficiency in the colon and small intestine and no colitis under basal conditions. a Metrnl mRNA expression in various tissues. WAT white adipose tissue. $n=8 .{ }^{*} P<0.05$. b Colon histological staining. Bar, $200 \mu \mathrm{m}$. c, d IL-6 and IL-4 mRNA expression and TNF- $\alpha$ protein levels in the colon. 
$3 \%$ DSS group and that all mice died after 10 days of dosing. In the $1 \%$ DSS group, no mice died during the experimental period. There were no significant changes in body weight loss of mice in the $1 \%$ DSS group, while the body weight of mice in the $3 \%$ DSS group was significantly reduced on the 5th day (Fig. 2b). In addition, the $1 \%$ DSS group showed no significant changes in gross parameters such as disease activity index and colon length, whereas the 3\% DSS group had increased disease activity index and shortened colon length when compared with those in the control group (Fig. 2c, d). Histological morphology exhibited the phenotypes of colitis with obvious tissue erosion in the 3\% DSS group (Fig. 2e). Moreover, the expression of Metrnl was comparable between the 3\% DSS and control groups. Therefore, we selected 3\% DSS and 5 days of dosing as the experimental conditions for subsequent experiments.

Metrnl deficiency exacerbates the symptoms and colon inflammation in DSS-induced colitis

After drinking 3\% DSS water for 5 days, both WT and Metrnl $^{-1-}$ mice developed severe illness characterized by the presence of sustained weight loss, bloody diarrhea, and severe colon inflammation, and these characteristics were associated with hyperemia, ulceration, and bowel wall thickening leading to further reduction in colon length. During this process, Metrnl ${ }^{-/-}$

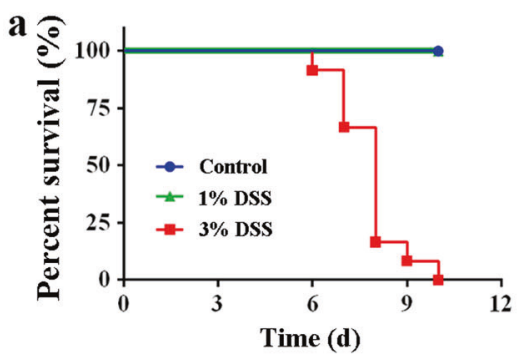

d
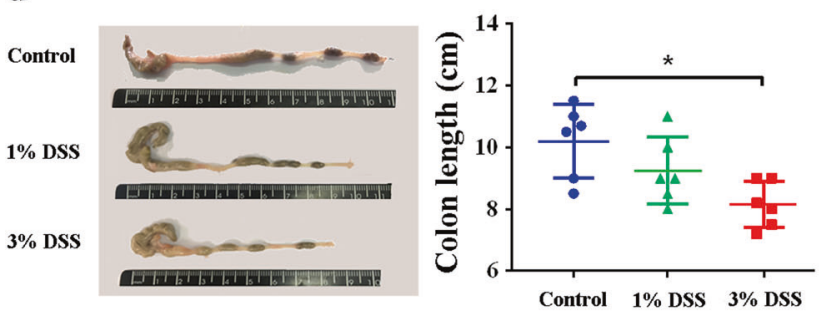

b

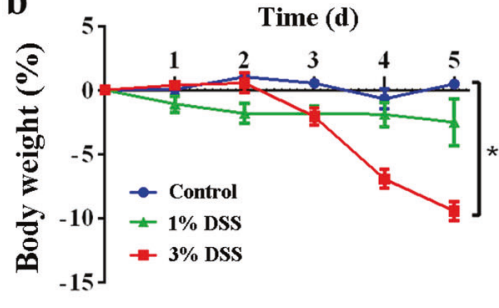

e

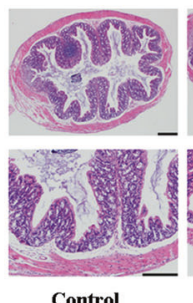

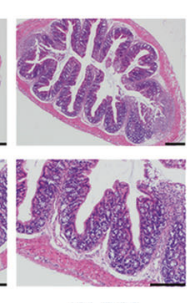

$1 \%$ DSS c

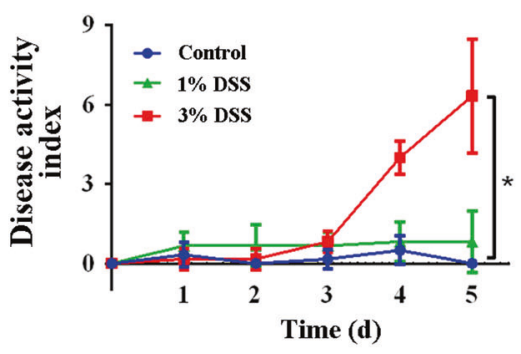

f

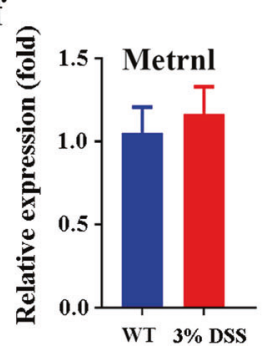

Fig. 2 Consuming 3\% DSS drinking water for 5 days induces ulcerative colitis in wild-type mice. a Survival curve in mice treated with 3\% or $1 \%$ DSS drinking water for 10 days. $n=12$. b-e Body weight loss, disease activity index, colon length, and histological morphology after consuming $3 \%$ or $1 \%$ DSS drinking water for 5 days. $n=6$. ${ }^{*} P<0.05$. $f$ Metrnl mRNA expression in mice treated with $3 \%$ or drinking water.

a

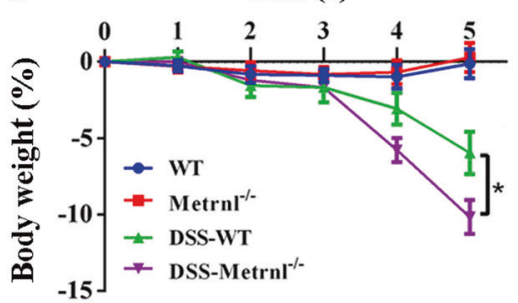

b

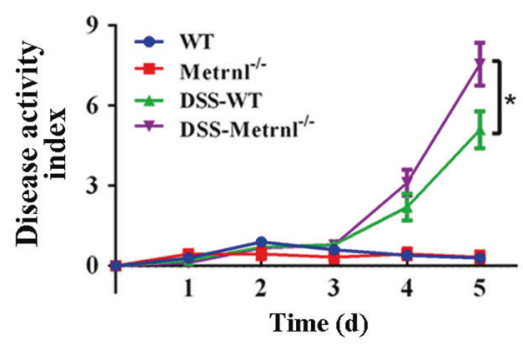

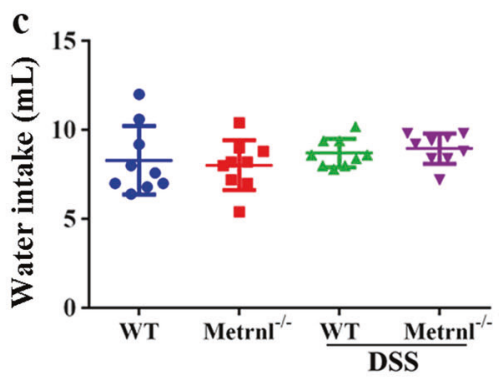

e

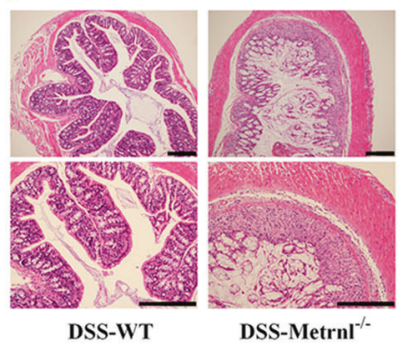

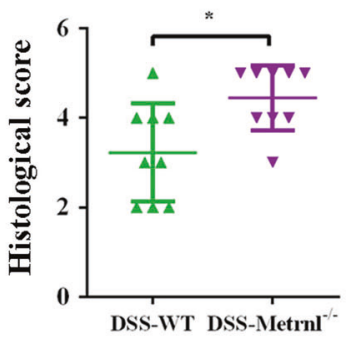

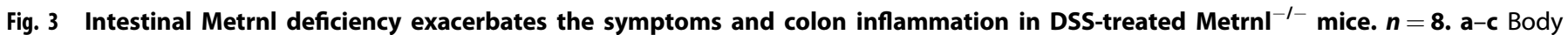
weight loss, disease activity index, and average drinking water intake at 5 days of 3\% DSS drinking water consumption. d, e Colon length and histological morphology after consuming 3\% DSS drinking water for 5 days. Bar, $200 \mu \mathrm{m} .{ }^{*} P<0.05$. 

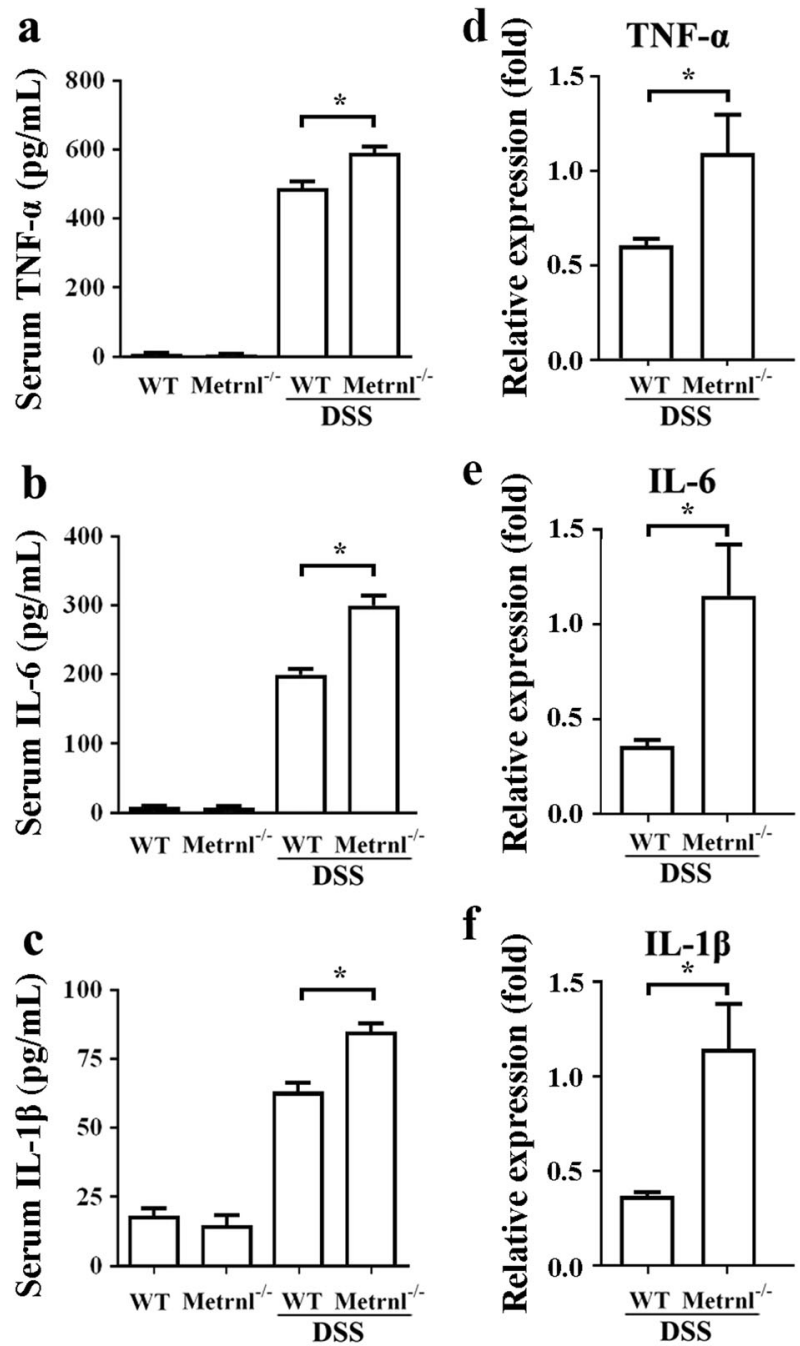

Fig. 4 Increased inflammatory factors in DSS-treated MetrnI ${ }^{-1-}$ mice. Serum levels of TNF- $\alpha(\mathbf{a}), \mathrm{IL}-6$ (b), and IL-1 $\beta$ (c) in Metrnl ${ }^{-1-}$ mice treated with $3 \%$ DSS for 5 days. $n=8$. ${ }^{*} P<0.05$. Relative mRNA expression of TNF- $\alpha(\mathbf{d})$, IL- $6(\mathbf{e})$, and IL-1 $\beta$ (f) in the colon of Metrnl $^{-I-}$ mice treated with $3 \%$ DSS for 5 days. $n=6 .{ }^{*} P<0.05$.

mice showed significantly worse symptoms of weight loss, bloody diarrhea, and colon inflammation, with aggravated inflammatory infiltration in mucosa and submucosa (Fig. 3a-e). To rule out that the above differences were caused by the intake of different amounts of $3 \%$ DSS by the mice, we also measured the drinking water of the two groups of mice. The results showed no significant differences between the two groups (Fig. 3c).

Metrnl deficiency increases the expression of inflammatory factors in DSS-induced colitis

To further verify the proinflammatory effects of Metrnl deficiency after DSS treatment, we tested inflammatory factors in mouse serum. Serum TNF- $\alpha$, IL- 6 , and IL- $1 \beta$ levels remained comparable between WT and Metrnl ${ }^{-1-}$ mice without DSS administration and were elevated in both groups after DSS administration. Moreover, TNF- $a$, IL-6, and IL-1 $\beta$ levels were significantly higher in DSStreated Metrnl ${ }^{-1-}$ mice than in WT mice (Fig. 4a-c). Furthermore, we investigated the levels of these inflammatory factors in the colon of Metrnl ${ }^{-1-}$ and WT mice treated with DSS. TNF-a, IL- 6 , and IL-1 $\beta$ mRNA levels were significantly higher in DSS-treated Metrnl $^{-/-}$mice than in WT mice (Fig. 4d-f).
Metrnl deficiency decreases the levels of autophagy induced by DSS in intestinal epithelial cells

We examined autophagy-related proteins and found that the level of autophagy in the Metrnl ${ }^{-1-}$ mice induced by 3\% DSS was significantly lower than that in the WT mice. Beclin-1 and LC3-II/I expression levels decreased and p62 expression levels increased in Metrnl $^{-1-}$ mice induced by 3\% DSS (Fig. 5a). To further verify that LC3-II was increased by enhancing the formation of autophagosomes, we measured autophagic flux by using the lysosomal inhibitor CQ. Consistent with the results from animal models, the in vitro results showed that Beclin-1 and LC3-II/I expression levels decreased and p62 expression levels increased in the Metrnl shRNA group compared with the control group under LPS treatment. Notably, CQ treatment abrogated the LPS-induced differences in autophagy levels between the Metrnl shRNA and control groups (Fig. 5b).

Further observations were obtained by transmission electron microscopy. The number of autophagosomes remained consistent between WT and Metrnl ${ }^{-/-}$mice without DSS administration and was increased in both groups after DSS administration. Moreover, the number of autophagosomes was lower in DSS-treated Metrnl $^{-1-}$ mice than in WT mice (Fig. 6a). To further verify the above results, we performed immunofluorescence on four groups of colon sections. The results showed that in the intestinal epithelial cell-specific marker CD326-labeled cells, the expression of LC3 in the $3 \%$ DSS-treated Metrnl ${ }^{-1-}$ mice was significantly lower than that in the 3\% DSS-treated WT mice (Fig. 6b).

Metrnl deficiency affects the autophagy-related AMPK-mTORp70S6K pathway in DSS-induced colitis

We further examined the effects of Metrnl on autophagy-related pathways. The results showed that DSS induced an increase in $p$ AMPK and a decrease in mTOR and p70S6K in WT mice. These effects were significantly reduced in $\mathrm{Metrnl}^{-1-}$ mice (Fig. 7), suggesting that the DSS-induced activation of the AMPK-mTORp70S6K pathway was partially blocked by Metrnl deficiency in the intestinal epithelium.

\section{DISCUSSION}

The present study demonstrates, for the first time, a protective role for Metrnl in murine DSS-induced colitis. This protection seems to be due to the regulation of Metrnl on the autophagy of intestinal epithelial cells in DSS-induced colitis. The conditional knockout of Metrnl in intestinal epithelial cells can downregulate autophagy levels in DSS-induced colitis through inhibition of the AMPK-mTOR-p70S6K pathway, thereby aggravating intestinal inflammation (Fig. 8).

Our lab screened for new adipokines in a global gene expression profiling of different adipose depots with bioinformatic methods in 2007. Metrnl was identified as a novel adipokine by our group [15]. Jorgensen et al. described Metrnl as a neurotrophic factor similar to Meteorin (Metrn) in 2012 [24]. Thus far, there are only a few studies on the function of Metrnl. Our previous study demonstrated that adipocyte Metrnl improves insulin sensitivity through the PPARY pathway in an autocrine/paracrine manner. Jorgensen et al. [24] reported the neurotrophic activity of Metrnl in neurite outgrowth and neuroblast migration in vitro and in the survival of spiral ganglion neurons in vivo. Watanabe et al. [25] reported that Metrnl is a latent process gene for cell differentiation and neurite extension length. Rao et al. [16] showed that Metrnl could promote browning of the subcutaneous and epididymal white adipose tissue.

There is a recent report on Metrnl ameliorating Crohn's disease, and it focused on the crosstalk of mesenteric adipose tissue and intestine. The study found that Metrnl administration attenuated mesenteric fat tissue lesions by promoting adipocyte function and differentiation partly through activating the STAT5/PPAR- $\gamma$ 
a

b

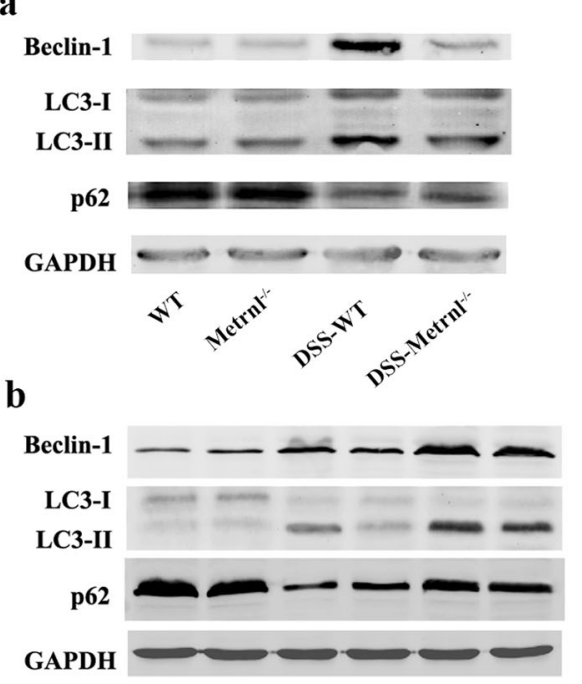

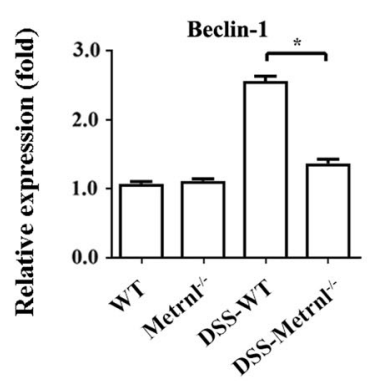

气્气

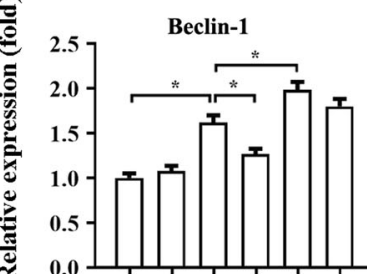

IsRNA - + - -+

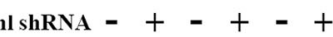

LPS $\quad-\quad+\quad+\quad+\quad+$ LPS $-\quad-+++$

$\mathrm{CQ} \quad-\quad-\quad-\quad-\quad+\quad+\quad \mathrm{CQ} \quad-\quad-\quad-++$
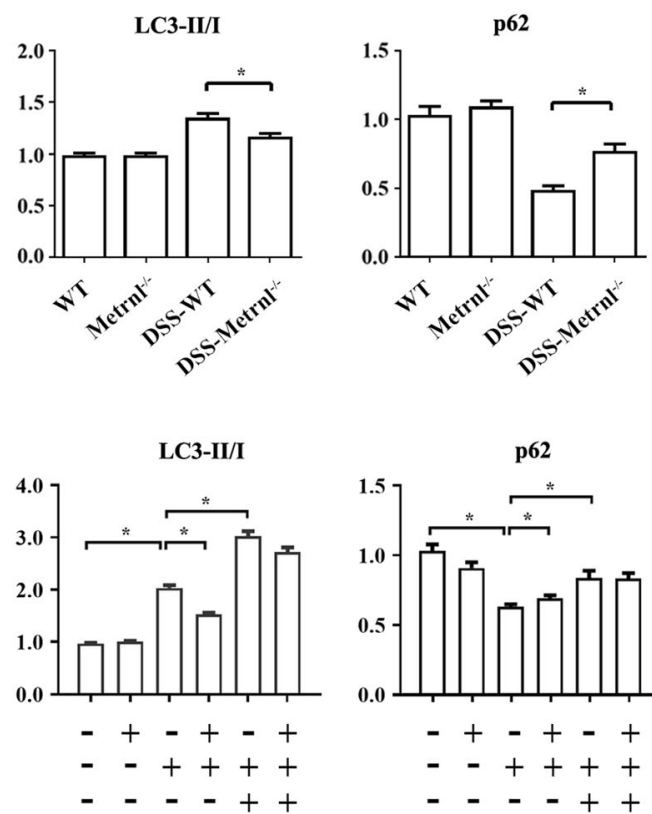

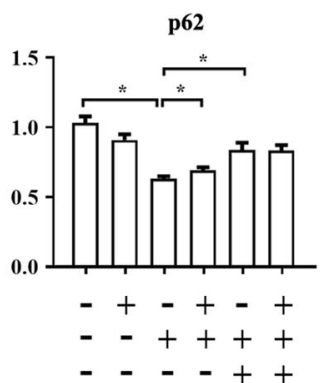

Fig. 5 Autophagy is downregulated by Metrnl deficiency in vivo and in vitro. a Representative Western blots and graphs showing the levels of Beclin-1, LC3-II/I, and p62 in DSS-treated Metrnl ${ }^{-/-}$mice. $n=8$. b Representative Western blots and graphs showing the levels of Beclin-1, LC3-II/I, and p62 in Caco-2 cells with knockdown of Metrnl. ${ }^{*} P<0.05$.

a

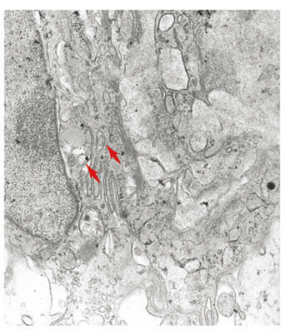

WT

b

WT

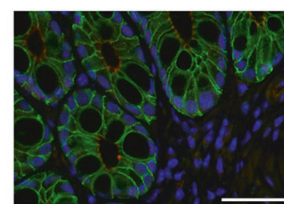

Metrnl $^{-/}$
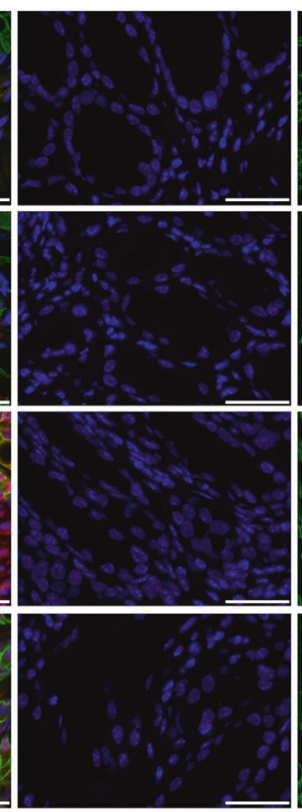

DAPI

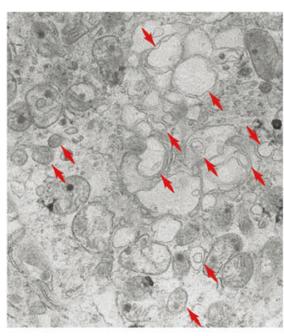

DSS-WT
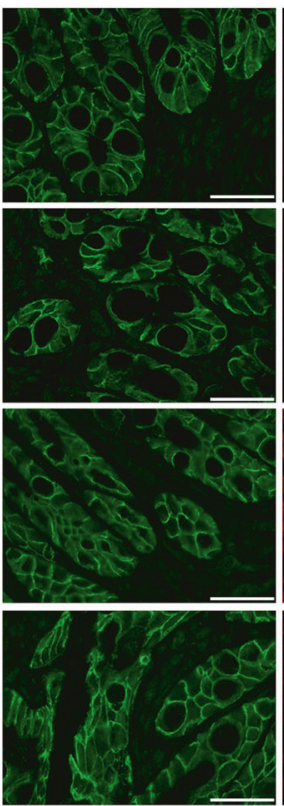

CD326

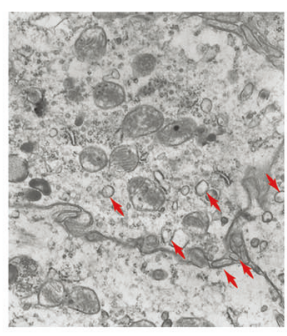

DSS-Metrnl ${ }^{-/}$
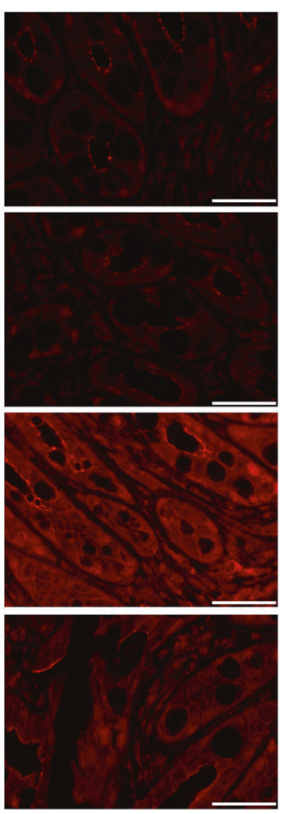

LC3

Fig. 6 Autophagy is downregulated in DSS-treated Metrnl ${ }^{-1-}$ mice. a Transmission electron micrograph of colon sections showing the ultrastructure of autophagosomes (red arrow). Original magnification $\times 12,000$. b Representative images of immunofluorescence staining of colon sections for DAPI (blue, nuclei indicator), LC3 (red, autophagy-related protein), and CD326 (green, epithelial marker). Original magnification $\times 800$. Bar, $50 \mu \mathrm{m}$. 

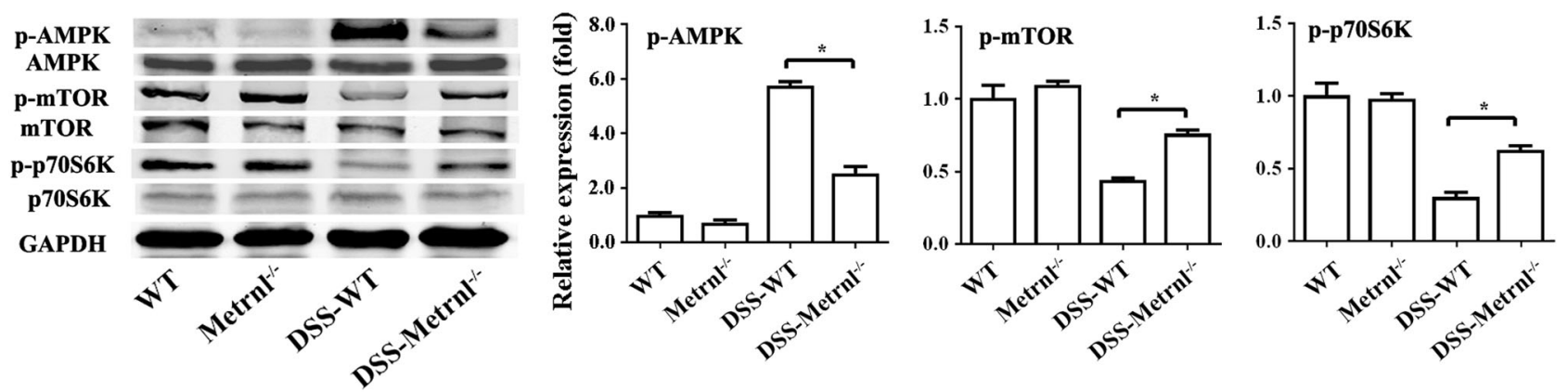

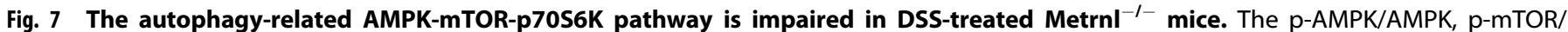
mTOR, and p-p70S6K/p70S6K ratios were determined by Western blots. $n=8$. ${ }^{*} P<0.05$.

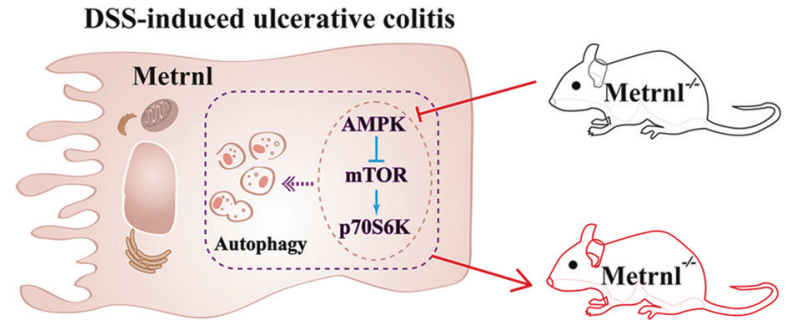

Epithelial cells

Fig. 8 Diagram showing the proposed pathway for the role of intestinal epithelial Metrnl deficiency in aggravating DSS-induced ulcerative colitis by reducing autophagy via the AMPK-mTORp70S6K pathway.

signaling pathway [18]. Although their research is different from ours, it indicated that Metrnl could truly affect inflammatory bowel disease; more directly, the administration of Metrnl has a protective effect on inflammatory bowel disease.

With the deepening of research on inflammatory bowel disease in recent years, there have been an increasing number of reports of autophagy affecting intestinal inflammation. In particular, inflammatory bowel disease susceptibility gene studies have revealed that multiple autophagy-related genes and their single nucleotide polymorphism sites, such as ATG16L1, NOD2, and IRGM, are associated with the pathogenesis of inflammatory bowel disease [26-29]. Moreover, currently, there are no reports about Metrnl and autophagy; therefore, we tested the role of Metrnl in autophagy-related proteins in DSS-induced colitis mice. To further clarify whether LC3-II was increased by enhancing the formation of autophagosomes or by decreasing the degradation of autolysosomes, autophagic flux was measured after the addition of $C Q$, a lysosomal blocker, to inhibit the formation of autophagosomes. Unsurprisingly, CQ increased autophagy in both the Metrnl shRNA and control groups. In addition, CQ abrogated the LPS-induced differences in autophagy levels between the Metrnl shRNA and control groups, suggesting that LC3-II increased due to the enhanced formation of autophagosomes instead of the decreased degradation of autolysosomes.

For the related mechanism of autophagy, current studies indicate that the AMPK-mTOR-p70S6K pathway is an important activating signaling pathway [30,31]. AMPK is an AMP-activated serine/threonine kinase that plays an important regulatory role in the metabolic process. AMP and ADP inhibit AMPK dephosphorylation and promote AMPK activation, thereby activating AMPK-dependent signaling pathways [32]. It has been reported that the activation of the AMPK-mTOR-p70S6K signaling pathway can significantly induce or increase autophagy levels in a variety of cells and diseases [33, 34]. Consistent with those

reports, in DSS-induced colitis, autophagy is induced by DSS in WT mice at least partially through this signaling pathway $[35,36]$.

There are shortcomings of this study. First, the protective function of autophagy on colitis is still under debate, and whether autophagy mediates the beneficial effects of Metrnl in colitis is not proven. Second, the signaling pathways activating autophagy are multiple and complex. In addition to the AMPK-mTOR-p70S6K signaling pathway, there are also other pathways, such as the $\mathrm{PI}$ KK/Akt/mTOR pathway, that can trigger autophagy but are not examined in the present study [7,37]. These deficiencies need to be clarified in future research.

In summary, the present study shows that Metrnl deficiency aggravates DSS-induced colitis, which is associated with the regulation of autophagy by Metrnl through the AMPK-mTORp70S6K pathway. These findings reveal that Metrnl is a potential therapeutic target for ulcerative colitis.

\section{ACKNOWLEDGEMENTS}

This study was supported by grants from the National Natural Science Foundation of China (No. 81803541 and No. 81730098).

\section{AUTHOR CONTRIBUTIONS}

SLZ and ZYL performed most of the experiments and data analyses and wrote the paper. DSW, TYX, MBF, and MHC performed some experiments and data analyses. CYM designed the study, performed data analysis, and wrote and revised the paper.

\section{ADDITIONAL INFORMATION}

Competing interests: The authors declare no competing interests.

\section{REFERENCES}

1. Ghosh S, Daperno M. Topical therapy in ulcerative colitis: always a bridesmaid but never a bride? Gastroenterology. 2015;148:701-4.

2. Ordas I, Eckmann L, Talamini M, Baumgart DC, Sandborn WJ. Ulcerative colitis. Lancet. 2012;380:1606-19.

3. Yan YX, Shao MJ, Qi Q, Xu YS, Yang XQ, Zhu FH, et al. Artemisinin analogue SM934 ameliorates DSS-induced mouse ulcerative colitis via suppressing neutrophils and macrophages. Acta Pharmacol Sin. 2018;39:1633-44.

4. Ford AC, Moayyedi P, Hanauer SB. Ulcerative colitis. Br Med J. 2013;346:f432.

5. Mowat C, Cole A, Windsor A, Ahmad T, Arnott I, Driscoll R, et al. Guidelines for the management of inflammatory bowel disease in adults. Gut. 2011;60:571-607.

6. Liu CZ, Li FY, Lv XF, Ma MM, Li XY, Lin CX, et al. Endophilin A2 regulates calciumactivated chloride channel activity via selective autophagy-mediated TMEM16A degradation. Acta Pharmacol Sin. 2019; https://doi.org/10.1038/s41401-019-0298-5. [Epub ahead of print]

7. Ding R, Wang X, Chen W, Li Z, Wei AL, Wang QB, et al. WX20120108, a novel IAP antagonist, induces tumor cell autophagy via activating ROS-FOXO pathway. Acta Pharmacol Sin. 2019;40:1466-79.

8. Yu $\mathrm{P}$, Wang HY, Tian M, Li AX, Chen XS, Wang XL, et al. Eukaryotic elongation factor- 2 kinase regulates the cross-talk between autophagy and pyroptosis in 
doxorubicin-treated human melanoma cells in vitro. Acta Pharmacol Sin. 2019;40:1237-44.

9. Nakahira K, Haspel JA, Rathinam VA, Lee SJ, Dolinay T, Lam HC, et al. Autophagy proteins regulate innate immune responses by inhibiting the release of mitochondrial DNA mediated by the NALP3 inflammasome. Nat Immunol. 2011;12:222-30.

10. Netea-Maier RT, Plantinga TS, van de Veerdonk FL, Smit JW, Netea MG. Modulation of inflammation by autophagy: consequences for human disease. Autophagy. 2016;12:245-60.

11. Shao BZ, Han BZ, Zeng YX, Su DF, Liu C. The roles of macrophage autophagy in atherosclerosis. Acta Pharmacol Sin. 2016;37:150-6.

12. Macias-Ceja DC, Cosin-Roger J, Ortiz-Masia D, Salvador P, Hernandez C. Stimulation of autophagy prevents intestinal mucosal inflammation and ameliorates murine colitis. Br J Pharmacol. 2017;174:2501-11.

13. Zheng SL, Li ZY, Song J, Liu JM, Miao CY. Metrnl: a secreted protein with new emerging functions. Acta Pharmacol Sin. 2016;37:571-9.

14. Li ZY, Song J, Zheng SL, Fan MB, Guan YF, Qu Y, et al. Adipocyte Metrnl antagonizes insulin resistance through PPARgamma signaling. Diabetes. 2015;64:4011-22.

15. Li ZY, Zheng SL, Wang $P, X u$ TY, Guan YF, Zhang YJ, et al. Subfatin is a novel adipokine and unlike Meteorin in adipose and brain expression. CNS Neurosci Ther. 2014;20:344-54.

16. Rao RR, Long JZ, White JP, Svensson KJ, Lou J, Lokurkar I, et al. Meteorin-like is a hormone that regulates immune-adipose interactions to increase beige fat thermogenesis. Cell. 2014;157:1279-91.

17. Li ZY, Fan MB, Zhang SL, Qu Y, Zheng SL, Song J, et al. Intestinal Metrnl released into the gut lumen acts as a local regulator for gut antimicrobial peptides. Acta Pharmacol Sin. 2016;37:1458-66.

18. Zuo L, Ge S, Ge Y, Li J, Zhu B, Zhang Z, et al. The adipokine Metrnl ameliorates chronic colitis in IL-10 $10^{-/-}$mice by attenuating mesenteric adipose tissue lesions during spontaneous colitis. J Crohns Colitis. 2019;13:931-41.

19. Hu G, Liu J, Zhen YZ, Xu R, Qiao Y, Wei J, et al. Rhein lysinate increases the median survival time of SAMP10 mice: protective role in the kidney. Acta Pharmacol Sin. 2013;34:515-21.

20. Wang $P, X u$ TY, Wei $K$, Guan YF, Wang $X, X u$ H, et al. ARRB1/beta-arrestin-1 mediates neuroprotection through coordination of BECN1-dependent autophagy in cerebral ischemia. Autophagy. 2014;10:1535-48.

21. Zheng SL, Li ZY, Zhang Z, Wang DS, Xu J, Miao CY. Evaluation of two commercial enzyme-linked immunosorbent assay kits for the detection of human circulating Metrnl. Chem Pharm Bull. 2018;66:391-98.

22. Tun X, Yasukawa K, Yamada K. Involvement of nitric oxide with activation of Tolllike receptor 4 signaling in mice with dextran sodium sulfate-induced colitis. Free Radic Biol Med. 2014;74:108-17.

23. Islam MS, Kusakabe $M$, Horiguchi $K$, lino $S$, Nakamura $T$, Iwanaga $K$, et al. PDGF and TGF-beta promote tenascin-C expression in subepithelial myofibroblasts and contribute to intestinal mucosal protection in mice. $\mathrm{Br} J$ Pharmacol. 2014;171:375-88.

24. Jorgensen JR, Fransson A, Fjord-Larsen L, Thompson LH, Houchins JP, Andrade N, et al. Cometin is a novel neurotrophic factor that promotes neurite outgrowth and neuroblast migration in vitro and supports survival of spiral ganglion neurons in vivo. Exp Neurol. 2012;233:172-81.

25. Watanabe K, Akimoto Y, Yugi K, Uda S, Chung J, Nakamuta S, et al. Latent process genes for cell differentiation are common decoders of neurite extension length. J Cell Sci. 2012;125:2198-211.

26. Saitoh T, Fujita N, Jang MH, Uematsu S, Yang BG, Satoh T, et al. Loss of the autophagy protein Atg16L1 enhances endotoxin-induced IL-1beta production. Nature. 2008;456:264-8.

27. Cadwell K, Liu JY, Brown SL, Miyoshi H, Loh J, Lennerz JK, et al. A key role for autophagy and the autophagy gene Atg16/1 in mouse and human intestinal Paneth cells. Nature. 2008;456:259-63.

28. Travassos LH, Carneiro LA, Ramjeet M, Hussey S, Kim YG, Magalhaes JG, et al. Nod1 and Nod2 direct autophagy by recruiting ATG16L1 to the plasma membrane at the site of bacterial entry. Nat Immunol. 2010;11:55-62.

29. McCarroll SA, Huett A, Kuballa P, Chilewski SD, Landry A, Goyette $P$, et al. Deletion polymorphism upstream of IRGM associated with altered IRGM expression and Crohn's disease. Nat Genet. 2008;40:1107-12.

30. Tong X, Gu J, Song R, Wang D, Sun Z, Sui C, et al. Osteoprotegerin inhibit osteoclast differentiation and bone resorption by enhancing autophagy via AMPK/mTOR/ p70S6K signaling pathway in vitro. J Cell Biochem. 2019:120:1630-42.

31. Wang Z, Lai ST, Ma NY, Deng Y, Liu Y, Wei DP, et al. Radiosensitization of metformin in pancreatic cancer cells via abrogating the $\mathrm{G} 2$ checkpoint and inhibiting DNA damage repair. Cancer Lett. 2015;369:192-201.

32. Klionsky DJ, Abdelmohsen $\mathrm{K}$, Abe A, Abedin MJ, Abeliovich $\mathrm{H}$, Acevedo Arozena $A$, et al. Guidelines for the use and interpretation of assays for monitoring autophagy (3rd edition). Autophagy. 2016;12:1-222.

33. Wu A, Hu P, Lin J, Xia W, Zhang R. Activating cannabinoid receptor 2 protects against diabetic cardiomyopathy through autophagy induction. Front Pharmacol. 2018;9:1292.

34. Duan P, Hu C, Quan C, Yu T, Huang W, Chen W, et al. 4-Nonylphenol induces autophagy and attenuates mTOR-p70S6K/4EBP1 signaling by modulating AMPK activation in Sertoli cells. Toxicol Lett. 2017;267:21-31.

35. Wang $P$, Guan YF, Du H, Zhai QW, Su DF, Miao CY. Induction of autophagy contributes to the neuroprotection of nicotinamide phosphoribosyltransferase in cerebral ischemia. Autophagy. 2012;8:77-87.

36. Wang P, Shao BZ, Deng Z, Chen S, Yue Z, Miao CY. Autophagy in ischemic stroke. Prog Neurobiol. 2018;163-164:98-117.

37. Zhang $H$, Gong $Y$, Wang $Z$, Jiang $L$, Chen $R$, Fan $X$, et al. Apelin inhibits the proliferation and migration of rat PASMCs via the activation of PI3K/Akt/mTOR signal and the inhibition of autophagy under hypoxia. J Cell Mol Med. 2014;18:542-53. 